\title{
Modular Input-Series-Input-Parallel Output-Series DC/DC Converter Control with Fault Detection and Redundancy
}

\author{
Yiqing. Lian*, D. Holliday, G.P. Adam, S.J. Finney \\ Department of Electronic and Electrical Engineering \\ University of Strathclyde, Glasgow, UK \\ * yiqing.lian@strath.ac.uk
}

\begin{abstract}
A novel high-power modular input-series-inputparallel output-series connected DC/DC converter for mediumvoltage application is proposed. Emphasis has been placed on power sharing control to compensate parameter mismatches and achieve equal power distribution between modules. Converter control is extended to achieve fault-tolerant operation by exploiting modularity to provide redundancy in the event of any failure. The proposed control scheme is validated through application-level simulations and scaleddown experiments to testify the reliability of the proposed control for ensuring power sharing between modules under a range of operating conditions. The results validate the proposed converter and associated control scheme indicating this to be a promising topology for high-power medium-voltage applications.
\end{abstract}

Keywords-High-power DC/DC converter, Redundancy, Small-signal modelling, Input-series-input-parallel Output-series

\section{INTRODUCTION}

Studies have shown that medium-voltage DC (MVDC) connection may be advantageous for long cable networks, such as the collection grid of offshore wind farms. In such cases, DC connection has the potential to deliver lower system level losses through the elimination of AC charging current and better utilization of cable capacity. Reduction in system volume and weight may also be achieved by the replacement of line-frequency transformers with mediumor high-frequency DC/DC converters [1-4]. Realizing the benefits of MVDC will require the use of high-capacity DC/DC converters capable of operating at the required network voltages, e.g. wind generator DC link voltage step up (e.g. from $5 \mathrm{kV}$ DC) to a level compatible with an efficient wide area network connection (e.g. $33 \mathrm{kV} \mathrm{DC}$ ). Although some high-efficiency DC/DC converters have been developed $[5,6]$, transfer of these technologies to the required network voltages is still a challenge since both the input and output of the DC/DC converter must operate above the voltage capability of existing power semiconductors. A solution to this problem is the modular connection of multiple transformer-coupled converters in which each module operates within the voltage rating of individual semiconductor devices, which can achieve highpower without compromising the efficiency and switching frequency. This paper proposes a multi-module converter consists of $n$ transformer-coupled full-bridge DC/DC modules, whose inputs are input-series-input-parallel connected, while the outputs are series connected, with unidirectional power flow capability.

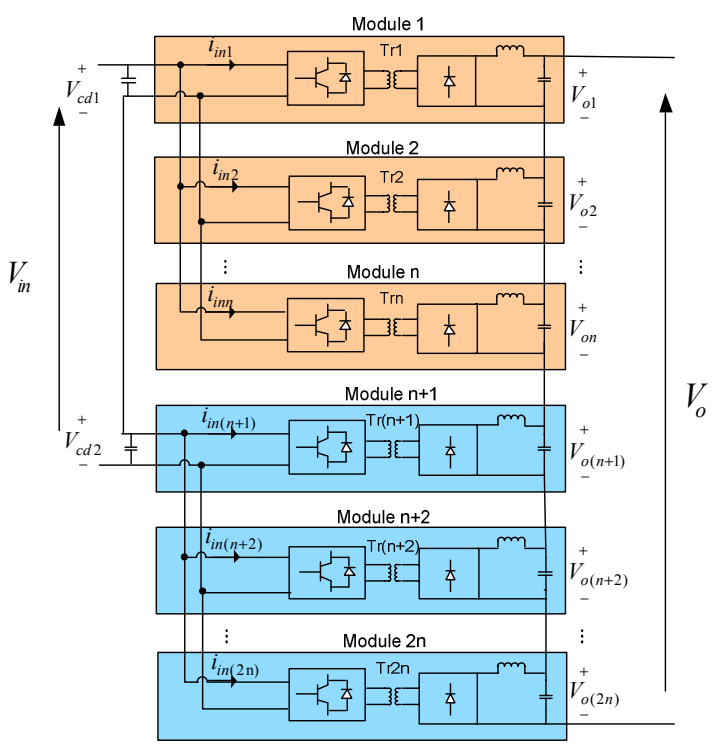

Fig.1 ISIPOS converter with full-bridge DC/DC modules

This configuration is well suited for offshore DC collection grid, where bi-directional power flow is not needed. In addition, it offers the potential for a more compact and lighter design [4, 7, 8]. Input-series-inputparallel connection of the power converters allows reduction of the current and voltage stresses in the power devices, thereby enabling device ratings and switching frequency to be optimized. With output-series connection, the turns-ratios of the isolation transformers can be reduced, leading to a reduction in leakage inductance. This proposed modular transformer-coupled DC/DC converter with input-seriesinput-parallel output-series (ISIPOS) connection presents a number of attractive properties: each module only contributes a small fraction of the total power and operates at voltages and currents that can be supported by available power semiconductors; the modular structure allows the 
converter to be re-configured and higher switching frequency can be achieved without significant design challenges; and redundant cells in this modular configuration provide fault ride through operation with a level of redundancy [8-10].

Fig. 1 shows the structure of a $2 n$-module ISIPOS DC/DC converter with two parallel arms (each has $n$ modules) being series connected, where the number of parallel and series connected modules can be extended to any number according to the application requirements. Reliable operation of the converter requires a dedicated controller that compensates for any mismatch in the passive components and active switching devices, and that ensures equal power sharing between the modules. The proposed multi-module converter has internal fault management capability similar to that of a modular multilevel converter (MMC), in that the controller can be designed to allow faulty modules to be bypassed in order to allow continued system operation without any performance degradation. Having introduced the proposed converter in Section I, small-signal modeling is introduced in Section II. The control topology for power sharing operation is described in Section III, and both simulation and experimental results are presented to confirm the viability of the control schemes. Simulation of fault-tolerant operation is presented in Section IV. Conclusions are presented in Section V.

\section{SMall Signal Modeling}

Although the DC/DC converter is a nonlinear system, the functions given here are used to facilitate controller design based on well-known linear feedback control techniques. The small-signal model of the four-module $(n=2$ in Fig.1) ISIPOS connected DC/DC converter, shown in Fig.2, builds upon a single module converter model [10] where $k_{1}, k_{2}, k_{3}$ and $k_{4}$ represent transformer turns ratios, $L_{r}$ represents the transformer leakage inductance, $L_{f 1}, L_{f 2}, L_{f 3}, L_{f 4}$, $C_{f 1}, C_{f 2}, C_{f 3}$ and $C_{f 4}$ are filter inductances and capacitances, and $\Delta v_{i n}$ is input voltage perturbation. Input voltage perturbation components for Modules 1 and 2, and for Modules 3 and 4 are $\Delta v_{c d 1}$ and $\Delta v_{c d 2}$ respectively, input current perturbations are $\Delta i_{i n 1}, \quad \Delta i_{i n 2}, \quad \Delta i_{i n 3}$ and $\Delta i_{i n 4}$ respectively, and filter inductor current and capacitor voltage perturbations are represented by $\Delta i_{l f 1}, \Delta i_{l f 2}, \Delta i_{l f 3}, \Delta i_{l f 4}, \Delta v_{o 1}$, $\Delta v_{o 2}, \Delta v_{o 3}, \Delta v_{o 4}$ respectively. $D_{e}$ is the effective duty ratio per module, duty ratio perturbations are represented by $\Delta d_{1}, \Delta d_{2}$, $\Delta d_{3}$, and $\Delta d_{4}$, and $\Delta d_{v 1}, \Delta d_{v 2}, \Delta d_{v 3}, \Delta d_{v 4}, \Delta d_{i 1}, \Delta d_{i 2}, \Delta d_{i 3}$ and $\Delta d_{i 4}$ represent perturbations of the duty ratio due to the input voltage and output current, as defined in (1)

$$
\left\{\begin{array}{c}
\Delta d_{v 1}=\Delta d_{v 2}=\frac{32 L_{r} D_{e} f_{s}}{k^{2} V_{i n} R_{o}} \Delta v_{c d 1} \\
\Delta d_{v 3}=\Delta d_{v 4}=\frac{32 L_{r} D_{e} f_{s}}{k^{2} V_{i n} R_{o}} \Delta v_{c d 2} \\
\Delta d_{i j}=-\frac{8 L_{r} f_{s}}{k V_{i n}} \Delta i_{l f j}
\end{array}\right.
$$

where $j=1,2,3$ and 4 .

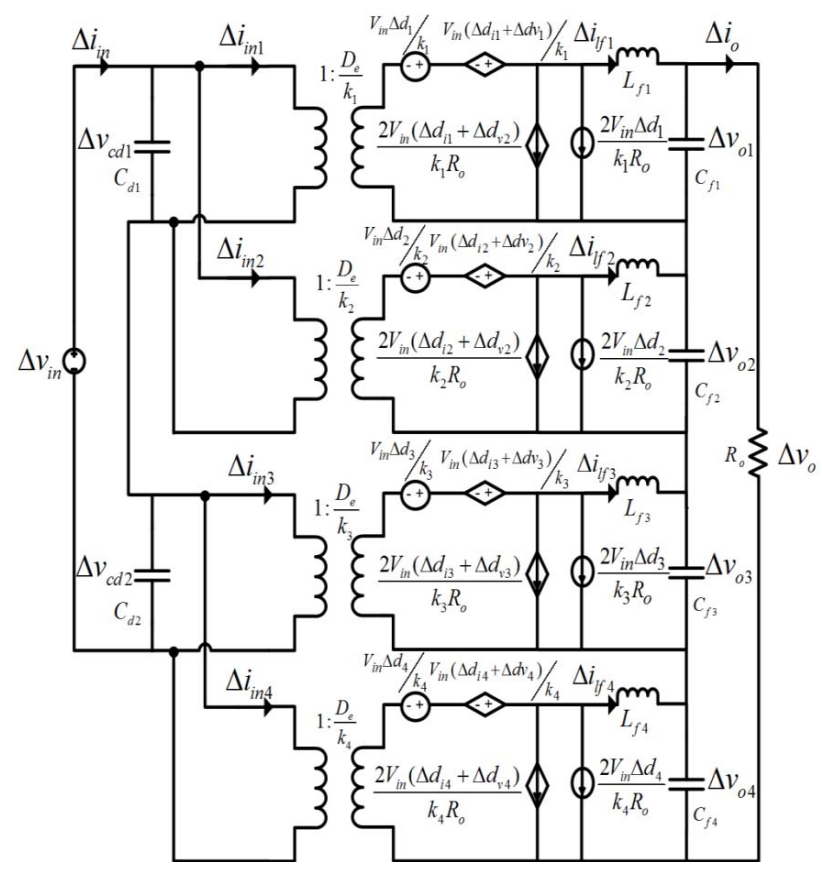

Fig.2 ISIPOS converter small-signal model

To simplify the analysis [11], four modules are assumed to have the same effective duty cycle, transformer turn ratio, and capacitor and inductor values. The corresponding equations, produced by application of KVL in Fig.2, are shown in (2).

$$
\left\{\begin{array}{l}
\frac{D_{e} \Delta v_{c d 1}}{k}+\frac{V_{i n}}{2 k}\left(\Delta d_{1}+\Delta d_{v 1}+\Delta d_{i 1}\right)=s \mathrm{~L}_{f} \Delta i_{l f 1}+\Delta v_{o 1} \\
\frac{D_{e} \Delta v_{c d 1}}{k}+\frac{V_{i n}}{2 k}\left(\Delta d_{2}+\Delta d_{v 2}+\Delta d_{i 2}\right)=s \mathrm{~L}_{f} \Delta i_{l f 2}+\Delta v_{o 2} \\
\frac{D_{e} \Delta v_{c d 2}}{k}+\frac{V_{i n}}{2 k}\left(\Delta d_{3}+\Delta d_{v 3}+\Delta d_{i 3}\right)=s \mathrm{~L}_{f} \Delta i_{l f 3}+\Delta v_{o 3} \\
\frac{D_{e} \Delta v_{c d 2}}{k}+\frac{V_{i n}}{2 k}\left(\Delta d_{4}+\Delta d_{v 4}+\Delta d_{i 4}\right)=s \mathrm{~L}_{f} \Delta i_{l f 4}+\Delta v_{o 4}
\end{array}\right.
$$

The relationship between output voltage and load current is derived [4] as (3).

$$
G_{\mathrm{o} v i}=\frac{\Delta v_{o}}{\Delta i_{o}}=\frac{4 L_{f} \mathrm{~s}+\frac{16 L_{r} f_{s}}{k^{2}}}{s^{2} L_{f} C_{f}+s\left(\frac{4 L_{f}}{R_{o}}+\frac{4 L_{r} f_{s} C_{f}}{k^{2}}\right)+1+\frac{16 L_{r} f_{\mathrm{s}}}{k^{2} R_{o}}}
$$

The relationship between output voltage and duty ratio can be represented as (4), and the transfer function between load current and duty ratio can be represented as (5), where $j=1,2,3,4$.

$$
\begin{gathered}
G_{\mathrm{ovd}}=\frac{\Delta v_{o}}{\Delta d_{j}}=\frac{V_{\text {in }}}{2 k\left[s^{2} L_{f} C_{f}+s\left(\frac{4 L_{f}}{R_{o}}+\frac{4 L_{r} f_{s} C_{f}}{k^{2}}\right)+1+\frac{16 L_{r} f_{\mathrm{s}}}{k^{2} R_{o}}\right]} \\
G_{\text {oid }}=\frac{\Delta i_{o}}{\Delta d_{j}}=\frac{V_{i n}}{2 k\left[s^{2} L_{f} C_{f} R_{o}+4 L_{f} s+\frac{4 L_{r} f_{s} C_{f} R_{o}}{k^{2}} s+R_{o}+\frac{16 L_{r} f_{\mathrm{s}}}{k^{2}}\right]}
\end{gathered}
$$


The relationship between modular voltage outputs and inductor currents can be represented as (6)

$$
\left\{\begin{array}{l}
\Delta v_{o 1}=g_{1} \Delta i_{l f 1}-g_{2}\left(\Delta i_{l f 2}+\Delta i_{l f 3}+\Delta i_{l f 4}\right) \\
\Delta v_{o 2}=g_{1} \Delta i_{l f 2}-g_{2}\left(\Delta i_{l f 1}+\Delta i_{l f 3}+\Delta i_{l f 4}\right) \\
\Delta v_{o 3}=g_{1} \Delta i_{l f 3}-g_{2}\left(\Delta i_{l f 1}+\Delta i_{l f 2}+\Delta i_{l f 4}\right) \\
\Delta v_{o 4}=g_{1} \Delta i_{l f 4}-g_{2}\left(\Delta i_{l f 1}+\Delta i_{l f 2}+\Delta i_{l f 3}\right)
\end{array}\right.
$$

where

$$
\left\{\begin{array}{l}
g_{1}=\frac{s C_{f} R_{o}+3}{s^{2} C_{f}^{2} R_{o}+4 s C_{f}} \\
g_{2}=\frac{1}{s^{2} C_{f}^{2} R_{o}+4 s C_{f}}
\end{array}\right.
$$

The relationship between the two input capacitor voltages and duty ratios is shown in (8)

$$
\left[\begin{array}{l}
\Delta v_{c d 1} \\
\Delta v_{c d 2}
\end{array}\right]=\left[\begin{array}{cc}
\frac{A(s)}{2} & -\frac{A(s)}{2} \\
-\frac{A(s)}{2} & \frac{A(s)}{2}
\end{array}\right]\left[\begin{array}{l}
\Delta d_{1} \\
\Delta d_{3}
\end{array}\right]
$$

where

$$
A(s)=-\frac{\frac{2 V_{i n}}{k R_{o}}\left(g_{2}+g_{1}+s L_{f}\right)+\frac{V_{i n}}{2 k}}{\frac{D_{e}}{k}+s C d \frac{k}{D_{e}}\left(g_{1}+s L_{f}+g_{2}\right)}
$$

The relationship between input currents and duty ratios is shown in (10)

$$
\begin{aligned}
& {\left[\begin{array}{l}
\Delta i_{i n 1} \\
\Delta i_{i n 2}
\end{array}\right]=\left[\begin{array}{cc}
\frac{B(s)}{2} & -\frac{B(s)}{2} \\
-\frac{B(s)}{2} & \frac{B(s)}{2}
\end{array}\right]\left[\begin{array}{l}
\Delta d_{1} \\
\Delta d_{2}
\end{array}\right]} \\
& {\left[\begin{array}{l}
\Delta i_{i n 3} \\
\Delta i_{i n 4}
\end{array}\right]=\left[\begin{array}{cc}
\frac{B(s)}{2} & -\frac{B(s)}{2} \\
-\frac{B(s)}{2} & \frac{B(s)}{2}
\end{array}\right]\left[\begin{array}{l}
\Delta d_{3} \\
\Delta d_{4}
\end{array}\right]}
\end{aligned}
$$

where

$$
\begin{gathered}
B(s)=D_{e}\left(\frac{2 V_{i n}}{R_{o}}+g_{3}-g_{4}\right) \\
\left\{\begin{array}{l}
g_{3}=\frac{a c-2 b c}{(a+b)(a-3 b)} \\
g_{4}=\frac{b c}{(a+b)(a-3 b)}
\end{array}\right. \\
\left\{\begin{array}{c}
a=s L_{f}+\frac{s C_{f} R_{o}+3}{s^{2} C_{f}^{2} R_{o}+4 s C_{f}}+\frac{4 L_{r} f_{s}}{k^{2}} \\
b=-\frac{1}{s^{2} C_{f}^{2} R_{o}+4 s C_{f}} \\
c=\frac{V_{i n}}{2 k}
\end{array}\right.
\end{gathered}
$$

\section{ISIPOS CONVERTER POWER BALANCING CONTROL}

\section{A. Power balancing control}

In order to ensure safe and reliable operation in the presence of internal mismatches and external transients, dedicated power balancing control is indispensable for ISIPOS DC/DC converters [11, 12].

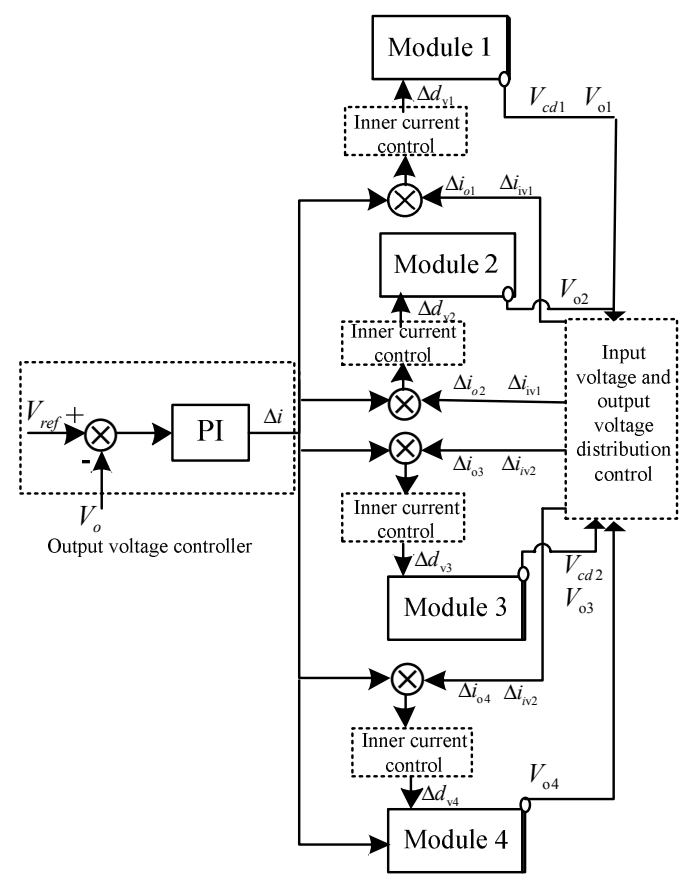

(a)

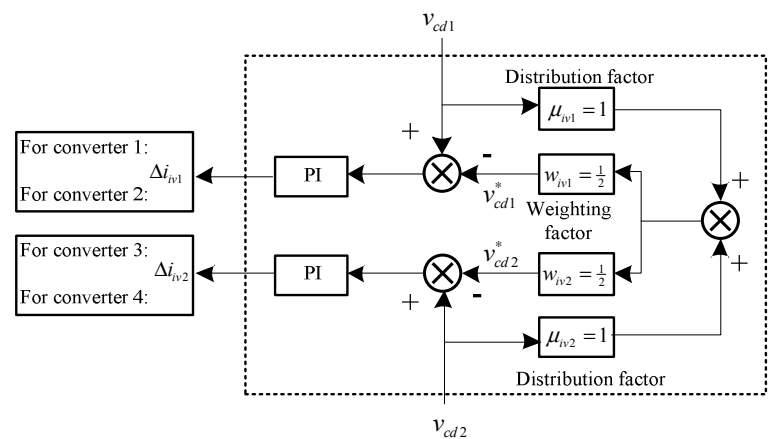

Input voltage sharing controller

(b)

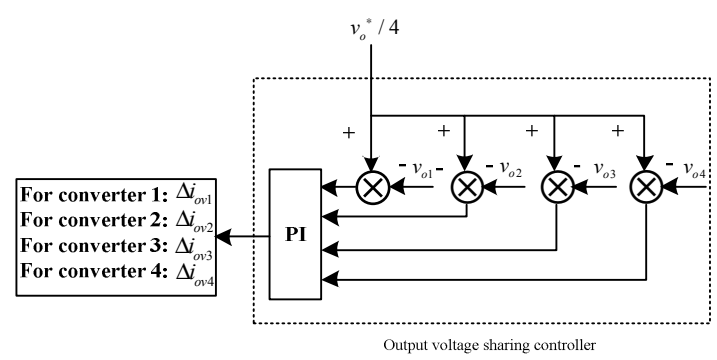

(c)

Fig. 3 Control scheme for the ISIPOS DC/DC converter (a) overall structure (b) input voltage sharing controller (c) output voltage sharing controller 
The proposed control system shown in Fig. 3 consists of four control loops: an output voltage control loop to control the load voltage, an output voltage sharing control loop to balance the modular output voltages, an input voltage sharing control loop to maintain the input capacitor voltages equal, and inner current loop that regulates the output inductance current of individual module and acts as safeguard against over-load.

As shown in Fig.3 (a), the output voltage controller contributes the main current signal $\Delta i$ to all modules based on (3), which is then modified by the power balancing loops. The control scheme provides power balancing control between all the modules using two control loops:

- Input voltage sharing loop

This maintains equal input voltages across the input capacitors in order to correct the differences between inputseries-output-series connected modules, e.g. Modules 1 and 3 . For input-series connected modules, the average sharing method applied to $v_{c d 1}$ and $v_{c d 2}$ is used to generate the input voltage reference signals. Therefore distribution factor $\mu_{i v 1}=\mu_{i v 2}=1$, and weighting functions $W_{i v 1}=W_{i v 2}=1 / 2$, as shown in Fig.3 (b). The input voltage sharing controller generates the current contributions $\Delta i_{i v 1}$ for Modules 1 and $2, \Delta i_{i v 2}$ for Modules 3 and 4.

\section{- $\quad$ Output voltage sharing loop}

This balances modular output voltages in order to maintain power balanced between the input-parallel-outputseries modules, e.g. Modules 1 and 2, or Modules 3 and 4. For input-parallel connected modules, $\Delta i_{o v 1}, \Delta i_{o v 2}, \Delta i_{o v 3}, \Delta i_{o v 4}$ are generated by the PI controller which eliminates the error between $v_{o}{ }^{*} / \mathrm{n}$, where $n=2$ in this case, and the output voltage from an individual module, as shown in Fig.3 (c).

Referring to Fig.3 (a), the current contributions produced by the three controllers are summed to provide the module current reference. Individual inner current control loops for filter inductor currents, which are capable of guaranteeing current performance, are added to obtain the duty cycle contributions from the PI current controller. Simulation and experimental results are presented in the next section to demonstrate its performance in the context of parameter mismatch and transience.

\section{B. Power balancing control simulation results}

The system parameters are listed in Table I. Note that some parameters are chosen to be different among the modules. Fig. 4 presents selected simulation results for a step change in load output voltage, with parameter mismatches between the modules. Fig.4 (a) shows DC output voltage performance as the output voltage reference changes to $33 \mathrm{kV}$ at time $t=0.1 \mathrm{~s}$ and then reduces to $30 \mathrm{kV}$ at $t=0.2 \mathrm{~s}$. Fig. 4 (b) and (c) show the corresponding modular output voltages and output current performances. These results show that the multi-module ISIPOS DC/DC converter can achieve equal power sharing between the modules with the proposed control scheme in the presence of mismatches, and that it outperforms a large single DC/DC converter based on a twolevel topology in terms of device stress, and based on an MMC topology in terms of dynamic performance and overall footprint resulting from its large capacitor requirement.

TABLE I: LIST OF PARAMETERS

\begin{tabular}{|c|c|}
\hline Parameter & Simulation Values \\
\hline DC/DC Converter Rated Power & $5 \mathrm{MW}$ \\
\hline Input DC Voltage & $5 \mathrm{kV}$ \\
\hline Output DC Voltage & $33 \mathrm{kV}$ \\
\hline Number of Modules & 10 \\
\hline PWM Carrier Frequency & $2.5 \mathrm{kHz}$ \\
\hline Input Capacitance & $\begin{array}{c}C_{c d 1}=1000 \mu \mathrm{F} \\
C_{c d 2}=1100 \mu \mathrm{F}\end{array}$ \\
\hline Transformer Turns Ratio & $\begin{array}{c}T_{1}=T_{3}=T_{5}=T_{7}=T_{9}=1: 1.2 \\
T_{2}=T_{4}=T_{6}=T_{8}=T_{10}=1: 1.3\end{array}$ \\
\hline Output Inductance & $\begin{array}{c}L_{1}=L_{3}=L_{5}=L_{7}=L_{9}=0.5 \mathrm{H} \\
L_{2}=L_{4}=L_{6}=L_{8}=L_{10}=0.55 \mathrm{H}\end{array}$ \\
\hline Output Capacitance & $\begin{array}{c}C_{1}=C_{3}=C_{5}=C_{7}=C_{9}=80 \mu \mathrm{F} \\
C_{2}=C_{4}=C_{6}=C_{8}=C_{10}=100 \mu \mathrm{F}\end{array}$ \\
\hline
\end{tabular}

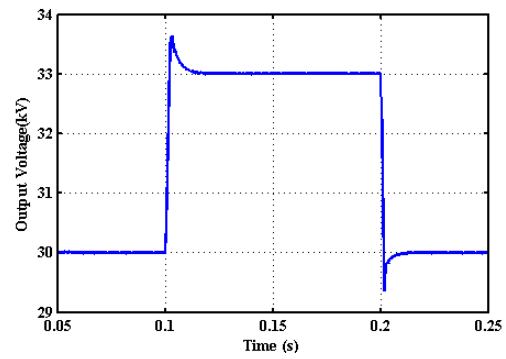

(a)

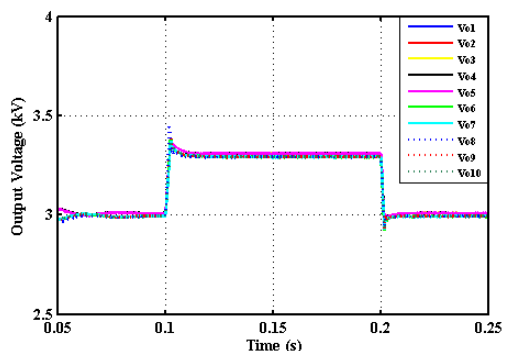

(b)

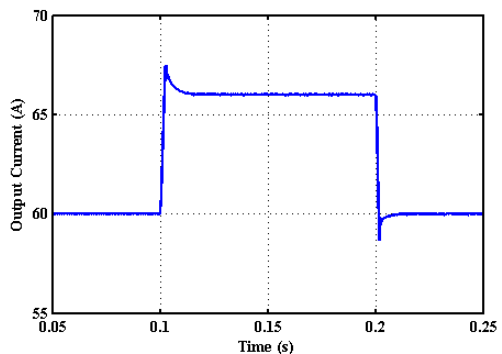

(c)

Fig.4 Simulation waveforms illustrating dynamic performance of the converter when a step change is applied to its DC output voltage reference (a) output voltage (b) modular output voltages (c) output current 


\section{Power balancing control experimental results}

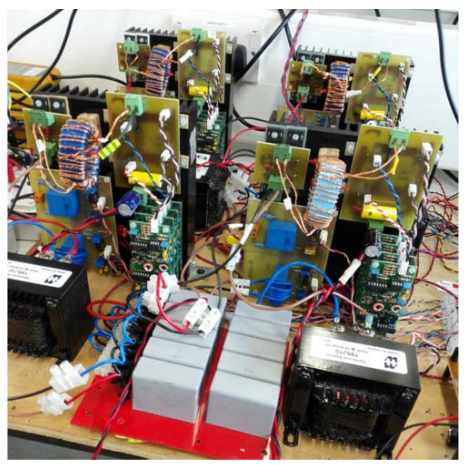

Fig. 5 Test rig for the ISIPOS connected DC/DC converter

Performance was validated by using the test rig shown in Fig.5. The test rig consists of four ISIPOS connected modules (i.e where $n=2$ in Fig. 1 ) and where the main system parameters are as listed in Table II.

TABLE II: LIST OF PARAMETERS

\begin{tabular}{|c|c|}
\hline Parameter & Simulation Values \\
\hline DC/DC Converter Rated Power & $200 \mathrm{~W}$ \\
\hline Input DC Voltage & $40 \mathrm{~V}$ \\
\hline Output DC Voltage & $60 \mathrm{~V}$ \\
\hline Number of Modules & 4 \\
\hline PWM Carrier Frequency & $2.5 \mathrm{kHz}$ \\
\hline Input Capacitance & $\begin{array}{c}C_{c d 1}=45 \mu \mathrm{F} \\
C_{c d 2}=40 \mu \mathrm{F}\end{array}$ \\
\hline Transformer Turns Ratio & $\begin{array}{c}T_{1}=1: 1.4, T_{2}=1: 1.2 \\
T_{3}=1: 1.3, T_{4}=1: 1.2\end{array}$ \\
\hline Output Inductance & $\begin{array}{c}L_{1}=L_{3}=L_{5}=L_{7}=L_{9}=0.5 \mathrm{H} \\
L_{2}=L_{4}=L_{6}=L_{8}=L_{10}=0.55 \mathrm{H}\end{array}$ \\
\hline Output Capacitance & $\begin{array}{c}C_{1}=C_{2}=160 \mu \mathrm{F} \\
C_{3}=C_{4}=200 \mu \mathrm{F}\end{array}$ \\
\hline
\end{tabular}

Fig.6 shows selected experimental results for normal operation of the ISIPOS converter, with mismatches between module parameters as shown in Table II. Fig.6 (a) shows input capacitor voltages $V_{c d 1}$ and $V_{c d 2}$ during start-up, when the same duty cycle is applied to the modules. It can be seen that there is a difference between the two voltages. Fig.6 (b) shows the results obtained when employing the proposed controller to balance the input capacitor voltages and that, despite the parameter mismatches, input voltage sharing (IVS) is achieved. Fig.6 (c) shows that, initially, control action is not active and same duty cycle is applied to the modules, and that when control action is activated at $t=150 \mathrm{~ms}$, IVS is achieved. This shows the effectiveness of the proposed control in achieving power sharing between the modules. Fig.6 (d) shows output voltage and current during start-up, where output voltage is regulated to the desired steady-state value of $60 \mathrm{~V}$ after $5 \mathrm{~ms}$. Modular output voltage during start-up is shown in Fig.6 (e), whilst Fig.6 (f) shows modular output voltage during steady-state. Both figures highlight the output voltage sharing (OVS) ability of the proposed controller. It can be observed that the proposed control scheme successfully addresses the issue of mismatched modules by balancing the input capacitor voltages and modular output voltages.

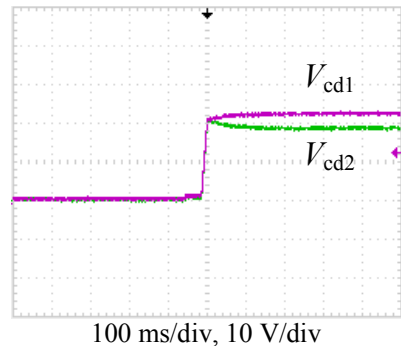

(a)

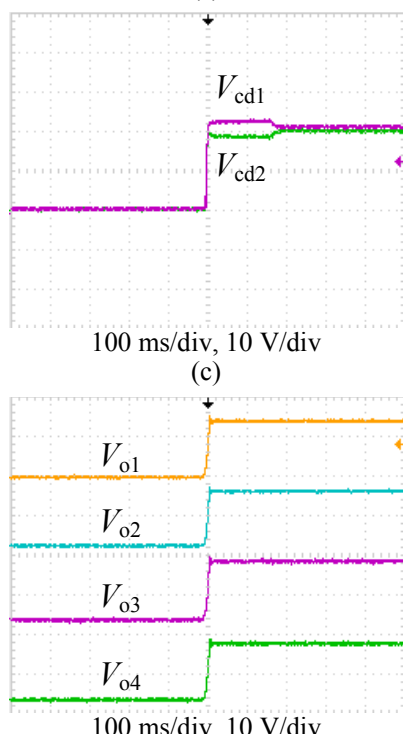

(e)

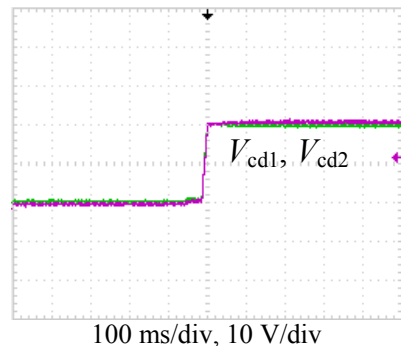

(b)

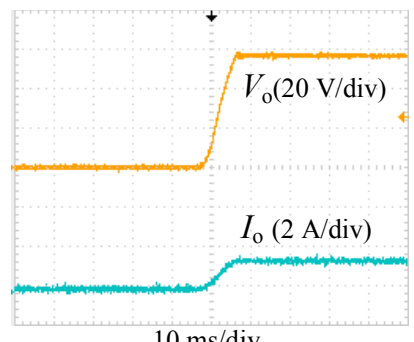

(d)

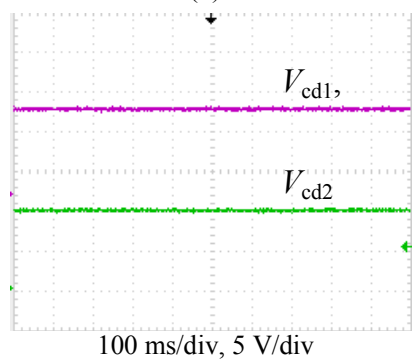

(f)
Fig.6 Experimental waveforms illustrating normal converter operation (a) input capacitor voltages without control (b) input capacitor voltages with control (c) input capacitor votlages without and with control (d) output votlage and current during start-up (e) modular output votlages during start-up (f) modular output votlages during steady-state

\section{ISIPOS CONVERTER FAULT DETECTION AND REDUNDANCY}

\section{A. Fault detection and redundancy control}

One of the merits of using this modular architecture is the increased reliability it provides for given level of redundancy. More modules than required by the load may be introduced to cater for module failure, so that each module operates at power level lower than the rated value under normal operation. When a fault occurs, the modularity feature allows a pre-designed control strategy to isolate the faulty module, and to reconfigure the converter to use the remaining healthy modules to supply the load. However, there is often a tradeoff between the extra cost incurred by the redundant modules and the impact of the failure [13].

Provision of redundancy can be illustrated by the converter in Fig.1, where $n=5$ and the faulty module is Module 5. Fault detection can be realized by monitoring the module output voltages, e.g. for the 10-module system shown in Fig.7, if the output voltage falls outside a pre- 
defined range, e.g. $V_{o} / 10.5<V_{o}<V_{o} / 9.5$, then a protection strategy is activated. The faulty module is isolated by blocking its front-end pulse width modulated H-bridge converter and bypassing its output diode bridge using a combination of a bypass switch and a bleed resistor to dissipate the energy stored in the filter capacitor. Following that, there are two ways of obtaining the fault-tolerant operation for this 10-module system:

The first method is to isolate Module 5, maintain voltage balance across the input capacitors, and to increase the output voltages of the remaining healthy modules (Module 1, 2,3 , and 4 ) in upper parallel-connected arm by changing the modular output voltage references from $V_{o} / 10$ to $V_{o} / 8$, to compensate the loss of Module 5. The output voltage references for the 5 modules in lower parallel-connected arm remain the same at $V_{o} / 10$. In doing this, the input currents to the modules in the upper parallel-connected arm are boosted to increase the input power.

For the second method, Module 5 and one module in the lower arm, e.g. Module 6, are isolated to maintain the symmetrical structure of the system. The output voltages of the remaining eight modules are boosted to track the new reference of $V_{o} / 8$.

In terms of stability, the symmetry offered by the second method simplifies the control action. However, more redundant modules are required in comparison to the first option, leading to additional cost. Therefore, the first option is adopted. Simulation results are presented in the next section to verify performance and fault-tolerant operation.

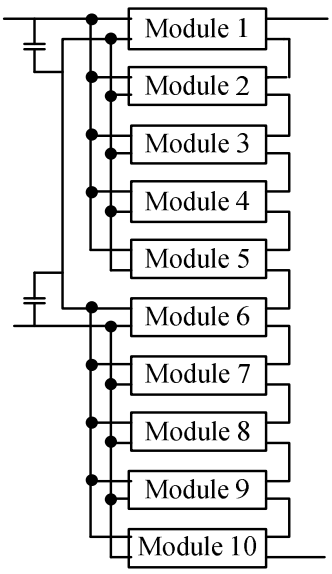

Fig.7 10-module ISIPOS DC/DC system

\section{B. Fault detection and redundancy control simulation results}

Fig. 8 presents selected simulation results for faulttolerant operation, with mismatches between module parameters as shown in Table I. A short-circuit fault is applied at the output of Module 5 at $t=75 \mathrm{~ms}$. It can be seen from Fig.8 (a) that converter output voltage $V_{o}$ recovers to its pre-fault value after a short time period. Following the fault, the faulty Module 5 is bypassed and its output becomes 0 . The output voltages of the remaining modules in the upper arm are boosted to compensate for the loss, and the modules in lower arm maintain their outputs. Fig.8 (c) shows the output current disturbance caused by the fault and that the recovery time is only a few milliseconds. Fig. 8 (d) proves the control loop can ensure input capacitor voltage balancing even under the fault condition. The results show that, with the mismatches in the system, the proposed control scheme provides fault-tolerant operation by isolating the faulty module and using the selected modules to compensate. This fault-tolerant operation proves the proposed converter has high reliability, which is an important factor in mediumvoltage applications.

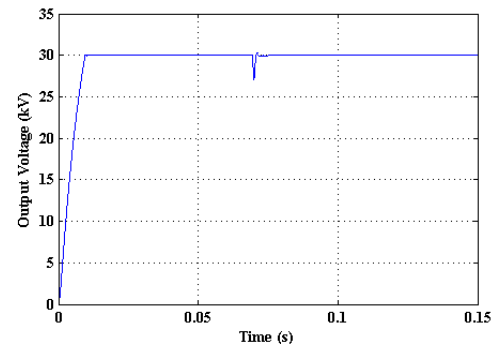

(a)

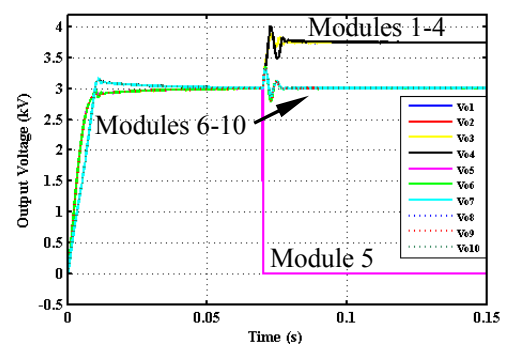

(b)

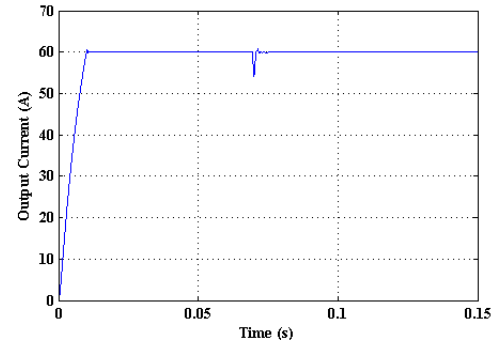

(c)

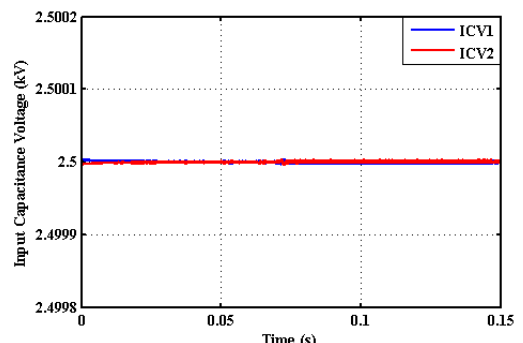

(d)

Fig.8 Simulation waveforms illustrating dynamic performance of the converter when a short-circuit fault is applied to the output of Module 5 (a) output voltage (b) modular output voltages (c) output current (d) input capacitor voltages 


\section{CONCLUSIONS}

A new high-power DC/DC converter for mediumvoltage applications is proposed, where full-bridge DC/DC converters are input-series-input-parallel output-series (ISIPOS) connected. Input voltage sharing (IVS) and output voltage sharing (OVS) control are proposed to achieve power sharing between the modules under conditions of module parameter mismatch. The power sharing control ability of the proposed control scheme is shown to effectively balance power distribution during dynamic operation. Simulation and experimental results to confirm this are presented based on a 10-module converter rated at $5 \mathrm{MW}$ and a 4-module scaled-down experimental test rig respectively. Reliability is an established metric for converter design, especially for high-power medium-voltage converters. Fault detection and redundancy can be easily employed in the proposed converter due to its modularity. This is verified through simulation of a 10 -module converter with one module failure and the results show that the system exploits $(n+1)$ redundancy. The proposed converter and control strategy can be extended to any number of modules and any allowable level of redundancy, making it a promising topology for high-power medium-voltage applications. Future work will incorporate additional modules into the converter, and will investigate application of $(n+1)$ redundancy to ISIPOS connected converters to show equal power sharing performance following a fault.

\section{ACKNOWLEDGMENT}

The authors gratefully acknowledge the support of the EPSRC Centre for Doctoral Training in Wind Energy Systems (EP/G037728/1) and the 'Underpinning Power Electronics 2012: Converters Theme' research programme (EP/K035096/1).

\section{REFERENCES}

[1] M. S. Carmeli, F. Castelli-Dezza, D. Rosati, G. Marchegiani, and M. Mauri, "MVDC connection of offshore wind farms to the transmission system," in Power Electronics Electrical Drives Automation and Motion (SPEEDAM), 2010 International Symposium on, Pisa, 2010, pp. 12011206.

[2] F. Deng, and C. Zhe, "Operation and Control of a DC-Grid Offshore Wind Farm Under DC Transmission System Faults," Power Delivery, IEEE Transactions on, vol. 28, no. 3, pp. 13561363, 2013.

[3] W. Chen, A. Q. Huang, C. Li, G. Wang, and W. $\mathrm{Gu}$, "Analysis and Comparison of Medium Voltage High Power DC/DC Converters for Offshore Wind Energy Systems," Power Electronics, IEEE
Transactions on, vol. 28, no. 4, pp. 2014-2023, 2013.

[4] Y. Lian, G. P. Adam, D. Holliday, and S. J. Finney, "Active power sharing in input-series-inputparallel output-series connected DC/DC converters," in Applied Power Electronics Conference and Exposition (APEC), 2015 IEEE, Charlotte, NC, 2015, pp. 2790-2797.

[5] C. Woo-Young, Y. Ju-Seung, and C. Jae-Yeon, "High efficiency dc-dc converter with high step-up gain for low PV voltage sources," in Power Electronics and ECCE Asia (ICPE \& ECCE), 2011 IEEE 8th International Conference on, Jeju, 2011, pp. 1161-1163.

[6] R. L. Steigerwald, R. W. De Doncker, and M. H. Kheraluwala, "A comparison of high-power DCDC soft-switched converter topologies," Industry Applications, IEEE Transactions on, vol. 32, no. 5, pp. 1139-1145, 1996.

[7] L. Yiqing, and S. J. Finney, "DC collection networks for offshore generation," in Renewable Power Generation Conference (RPG 2013), 2nd IET, Beijing, 2013, pp. 1-4.

[8] C. Wu, R. Xinbo, Y. Hong, and C. K. Tse, "DC/DC Conversion Systems Consisting of Multiple Converter Modules: Stability, Control, and Experimental Verifications," Power Electronics, IEEE Transactions on, vol. 24, no. 6, pp. 1463-1474, 2009.

[9] S. Chunwei, R. Zhao, L. Wangqing, and Z. Zheng, "A novel control strategy for input-parallel-outputseries inverter system," in Electrical Machines and Systems (ICEMS), 2011 International Conference on, Beijing, 2011, pp. 1-5.

[10] R. Xinbo, C. Wu, C. Lulu, C. K. Tse, Y. Hong, and Z. Tao, "Control Strategy for Input-Series\&OutputParallel Converters," Industrial Electronics, IEEE Transactions on, vol. 56, no. 4, pp. 1174-1185, 2009.

[11] S. Deshang, D. Kai, and L. Xiaozhong, "Duty Cycle Exchanging Control for Input-Series-OutputSeries Connected Two PS-FB DC-DC Converters," Power Electronics, IEEE Transactions on, vol. 27, no. 3, pp. 1490-1501, 2012.

[12] R. Xinbo, C. Lulu, and Z. Tao, "Control Strategy for Input-Series Output-Paralleled Converter," in Power Electronics Specialists Conference, 2006. PESC '06. 37th IEEE, Jeju, 2006, pp. 1-8.

[13] V. Choudhary, E. Ledezma, R. Ayyanar, and R. M. Button, "Fault Tolerant Circuit Topology and Control Method for Input-Series and OutputParallel Modular DC-DC Converters," Power Electronics, IEEE Transactions on, vol. 23, no. 1, pp. 402-411, 2008. 